\title{
Materials by design: Using architecture in material design to reach new property spaces
}

\author{
Lauren Montemayor, Victoria Chernow, and Julia R. Greer
}

\begin{abstract}
Mimicking the resilience offered by hard biomaterials, such as mollusk shells and beaks, has been among the most sought-after engineering pursuits. Technological advances in fabrication methods have provided pathways for using different materials to create architected structural metamaterials with hierarchy and length scales similar to those found in nature. Inspiration from nature has led to the creation of structural metamaterials, or nanolattices, with enhanced mechanical properties caused by hierarchical ordering at various length scales, ranging from angstroms and nanometers for the material microstructure to microns and millimeters for the macroscale architecture. The inherent periodicity and high surfacearea-to-volume ratios of nanolattices make them useful for a variety of applications, including photonics, photovoltaics, phononics, and electrochemical systems. This article provides an overview of current three-dimensional architected metamaterials, including their fabrication methods, properties, applications, and limitations.
\end{abstract}

\section{Introduction}

Materials with architected microstructures, such as butterfly wings and diatoms, are often found in nature. Structures on multiple length scales allow for a variety of novel nanoscale properties to propagate on to macroscopic structures. For example, the nanoscale architecture of a butterfly wing interacts with light to create iridescent, colorful wings, and smallscale ceramic building blocks are the key behind the unique material properties of nacre. ${ }^{1}$ Materials scientists and engineers have utilized the idea of architecture and hierarchy to create different kinds of novel metamaterials, taking inspiration from nature.

Architecture, here referred to an arrangement of beam elements into a three-dimensional (3D) structure such as a truss or lattice, has been used for many years to improve the efficiency of large engineered structures. Consider the Great Pyramid of Giza and the Eiffel Tower, two structures with vastly different architectures. The Great Pyramid is analogous to common bulk engineering materials, whereas the Eiffel Tower is analogous to a structural metamaterial, in which the lattice-based architecture spans length scales down to the material microstructure, which determines its strength. Structural metamaterials gain unique mechanical properties from the hierarchical ordering of length scales within the material, from the microstructure of the constituent material to large-scale structural ordering.

As the external dimensions of materials are reduced to the nanoscale, their mechanical behavior changes. ${ }^{14}$ These structural metamaterials have unique properties that stem from the linked behavior of the constituent material and the structure at small dimensions, rather than from either one independently.

Three-dimensional architecture and periodicity on the nano- and microscales allow novel control over properties such as the propagation of photons, phonons, and electrons through the structure. Photonic crystals ( $\mathrm{PhCs}$ ) are architected materials composed of repeating structural units with varying indices of refraction that selectively reflect light of wavelengths commensurate with their periodicity. $\mathrm{PhCs}$ underlie many striking coloration effects seen in natural systems. The iridescent colors found in opals, beetle scales, peacock feathers, and even the skin of certain cephalopods are the result of periodic geometries that can be as complex as the double gyroid and the kagome lattice or as relatively simple as close-packed spherical particles, cylinders, and lamellae. ${ }^{1,2}$ 
Similarly, phononic crystals (PnCs) are artificial periodic structures composed of elastic materials, in which mechanical waves within a specific frequency bandwidth are forbidden from propagating. This prohibited frequency range is termed the phononic bandgap (PnBG) and enables sound, and often heat, to be controlled, allowing for the creation of more efficient filters, waveguides, and resonant cavities.

The combination of geometry-including lattice type, topology, and scale-with material properties determines the ultimate behavior of architected materials. Beyond controlling wave propagation and mechanical properties, structural design and microstructural parameters are significant for many applications, including battery electrodes, supercapacitors, and other electrochemical (e.g., electrochromic) devices.

\section{Structural metamaterials}

Architecture has been used in a variety of engineering applications to create structures that carry load using a substantially lower amount of raw materials than those obtained using brick-and-mortar-like approaches. Macroscale cellular solids, such as the microlattice, have been studied experimentally, computationally, and theoretically, under the assumption that the properties of the constituent solids are constant, with the geometry being the primary tunable parameter. ${ }^{3-12}$ Structural metamaterials extend this concept of architecture to multiple length scales, even down to the material microstructure, on the order of nanometers, where materials exhibit size effects, thus making it possible to tune the properties of the constituent materials.

The so-called "material size effect" in mechanical properties that emerges at the nanoscale can manifest itself as a "smaller-is-stronger" effect, ${ }^{13-21}$ a "smaller-is-weaker" effect, ${ }^{21-23}$ or a brittle-to-ductile transition ${ }^{14,24-27}$ and can be exploited by employing micron- to nanometer-scale building blocks to create larger structures.

For example, stochastic foams, or foams whose pore distribution is random, such as nanoporous gold (shown in Figure 1), have been explored as a possible vehicle for propagating material size effects onto larger scales. ${ }^{28}$ All foams ultimately suffer from a rapid drop in strength and stiffness with density because the fabrication methodologies limit their geometries to being random.

Recently, various nanolattices with precisely controlled geometries that can be used to create exceptionally lightweight, strong, and tough metamaterials have been developed. Control of the 3D lattice geometry has been made possible by advances in micro- and nanofabrication techniques, ranging from polymer waveguides, ${ }^{29-34}$ to microstereolithography, ${ }^{30}$ to direct-laser-writing two-photon lithography (DLW TPL). ${ }^{31-33}$ The following sections provide an overview of some of these fabrication techniques and the resulting 3D-architected materials. The mechanical properties and densities of these materials are summarized in Table $\mathbf{I}$.

\section{Microlattices prepared by polymer waveguide technology}

The microlattice shown at the right side of Figure $\mathbf{2}$ is a cellular solid that was developed by HRL with dimensions on the order of centimeters and absolute densities as low as $0.9 \mathrm{mg} / \mathrm{cm}^{3}$, which places it in the ultralightweight regime, defined as materials with densities below $10 \mathrm{mg} / \mathrm{cm}^{3}{ }^{35}$ This microlattice is composed of hollow metallic struts with widths of $100-500 \mu \mathrm{m}$ and lengths of 1-4 $\mathrm{mm}$; the wall thickness of the hollow cylindrical struts is on the order of 100-500 nm. ${ }^{35}$

Figure 2 shows a sample made using a polymer waveguide technique, a self-guided single-photon polymerization process using angled UV beams traveling through a mask that allows for fast throughput of macroscale sheets. ${ }^{29,34}$ In the subsequent fabrication steps, the polymer scaffold is coated with a thin solid film, such as NiP or metallic glass. ${ }^{24,36}$ Removal of the polymer leaves a fully hollow structure composed of the deposited material..$^{35,36,41}$ The waveguide technique allows easy adjustment of the angle and the number of bars connecting at a single node, illustrating the relationship between the mechanical properties and geometric parameters of ultralight hollow structures. ${ }^{34}$

This patterning technique can generate only certain unitcell geometries, all of which deform under an applied load by bending, rather than stretching, the lattice bars. ${ }^{42}$ This bendingmode deformation provides lower strength than stretchingmode deformation for any given density. ${ }^{8,43}$

\section{Microlattices prepared by microstereolithography}

Microarchitectures with dimensions similar to those of the microlattices just discussed have also been created using microstereolithography, shown in Figure 3, which is an additive process with lattices created in a layer-by-layer fashion similar to $3 \mathrm{D}$ printing. ${ }^{30}$ In contrast to the waveguide method, this technique is capable of producing a variety of different geometries, including the octet truss, which has sufficiently

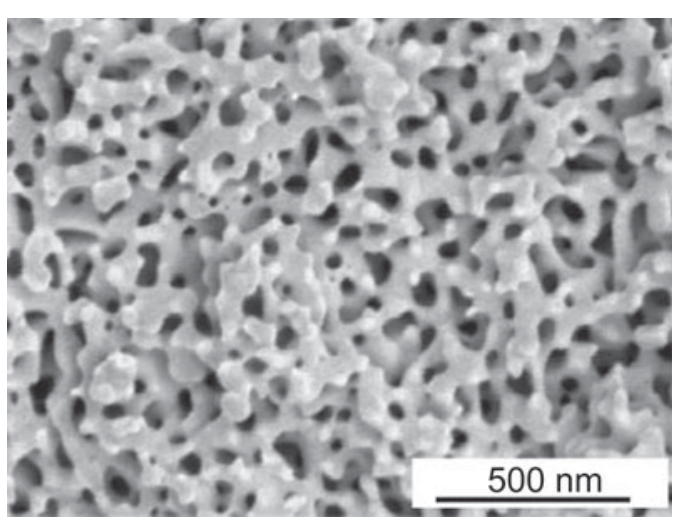

Figure 1. Representative image of nanoporous gold. Reproduced with permission from Reference 28. (C) 2005 Cambridge University Press. 
Table I. Summary of structural metamaterials in terms of fabrication method, relative density, strength, and modulus for the materials discussed in this article.*

\begin{tabular}{|c|c|c|c|c|c|c|}
\hline Lattice Type & Fabrication Method & Lattice Material & $\begin{array}{l}\text { Wall Thickness } \\
(\mathrm{nm})\end{array}$ & $\begin{array}{l}\text { Relative } \\
\text { Density } \rho\end{array}$ & $\begin{array}{l}\text { Strength } \sigma \\
(\mathrm{MPa})\end{array}$ & $\begin{array}{c}\text { Young's } \\
\text { Modulus } E(\mathrm{kPa})\end{array}$ \\
\hline $\begin{array}{l}\text { Microlattice (bending-, } \\
\text { stretching-dominated) }\end{array}$ & $\begin{array}{l}\text { Polymer wave-guide, } \\
\text { micro-stereolithography }\end{array}$ & $\begin{array}{l}\text { Polymer, hollow } \mathrm{Ni} \text {, } \\
\text { hollow } \mathrm{NiP} \text {, hollow/ } \\
\text { solid } \mathrm{Al}_{2} \mathrm{O}_{3}\end{array}$ & $\begin{array}{l}40-210 \mathrm{Al}_{2} \mathrm{O}_{3} \\
100-1000 \mathrm{NiP} \\
100-500 \mathrm{Ni}\end{array}$ & $10^{-4}-10^{-2}$ & $0.009-2.9$ & $10^{-1}-10^{8}$ \\
\hline $\begin{array}{l}\text { Nanolattice (bending-, } \\
\text { stretching-dominated) }\end{array}$ & DLW TPL & $\begin{array}{l}\text { Polymer, } \mathrm{Au}, \mathrm{Al}_{2} \mathrm{O}_{3} \text {, } \\
\text { polymer } / \mathrm{Al}_{2} \mathrm{O}_{3} \\
\text { composite }\end{array}$ & $\begin{array}{l}200-700 \mathrm{Au} \\
5-60 \mathrm{Al}_{2} \mathrm{O}_{3}\end{array}$ & $10^{-3}-10^{-1}$ & $10^{-1}-10^{2}$ & $1-10^{3}$ \\
\hline $\begin{array}{l}\text { Mesolattice (stretching- } \\
\text { dominated) }\end{array}$ & DLW TPL & $\mathrm{Cu}$ & - & $0.4-0.8$ & $50-350$ & - \\
\hline
\end{tabular}

Note: DLW TPL, direct-laser-writing two-photon lithography.

*Data compiled from References 24, 30, and 35-40.

high nodal connectivity to be stretching-dominated. ${ }^{7-9}$ Under uniaxial compression, hollow $\mathrm{NiP}$ or $\mathrm{Al}_{2} \mathrm{O}_{3}$ octet-truss microlattices have shown near agreement with the scaling of stiffness $(E)$ and strength $(\sigma)$ to the relative density $(\bar{\rho})$ predicted by cellular-solids theory, namely, $E \propto \bar{\rho}^{m}$ and $\sigma \propto \bar{\rho}^{n}$, with $m=n=1$. Uniaxial compression experiments gave values of $m=1.1$ and $n=1.2-2.7$ for octet-truss microlattices with relative densities of $\bar{\rho}=10^{-4}-10^{-2},{ }^{30}$ where relative density $(\bar{\rho})$ is the ratio of the volume contained within a unit cell to the volume of the unit cell. ${ }^{8,43}$ These octet trusses have higher strength than the HRL microlattices, which is attributed to the stretching-dominated architectures of the former. ${ }^{30,35}$

Hollow microlattices produced by either polymer waveguides or microstereolithograhy have wall thicknesses in the size regime where material size effects have been observed, but the dimensions of their unit cells are too large to observe or capitalize on the size effects because their deformation behaviors can be fully described by structural mechanics, which is independent of material length scales. For example, if the microlattices were scaled up in a self-similar geometry, the mechanisms of deformation would be consistent with those of the microlattices at smaller scales. These microlattices offer many novel properties that result from the interaction of various structural length scales, such as their recovery and energy-absorption capabilities. It would not be appropriate to classify them as structural metamaterials because their properties can be fully described by classical mechanics.

\section{Nanolattices prepared by direct-laser-writing two- photon lithography}

Nanolattices fabricated by two-photon lithography (TPL) enable a size reduction by three orders of magnitude from microlattices while still being amenable to coating and scaffold removal. These architected materials have dimensions down to the nanoscale, with typical strut lengths of 3-20 $\mu \mathrm{m}$, widths of $150-500 \mathrm{~nm}$, and wall thicknesses of 5-600 nm..$^{37,42,44,45}$ Unlike microlattices, the individual building blocks that comprise nanolattices are small enough that they cannot be resolved by the human eye. Their mechanical properties depend on a combination of size-dependent material properties and structural responses and cannot be predicted solely by scale-free continuum theories. ${ }^{37-39}$

Nanolattices were first fabricated from a specific polymer using DLW TPL developed by Nanoscribe GmbH. ${ }^{45}$ The two-photon method employs a 780-nm femtosecond pulsed laser focused into a small volume within the polymer (i.e., a voxel, or volume resolution element) with sufficient energy to cross-link the monomer and harden the material in that location through two-photon absorption. The ellipsoidal voxel can be rastered in three dimensions within a droplet of photoresist to create a polymer structure of arbitrary geometry, shown in Figure 4a..$^{31,32}$ A variety of existing thin-film deposition techniques lend themselves to the coating of these polymer nanolattices. ${ }^{38,40,42,44,45}$

For example, Montemayor et al. ${ }^{43}$ demonstrated the conformal sputtering of metals (Figure 4b), such as gold, onto different geometries of nanolattices as long as the relative density was below $\bar{\rho} \approx 4 \%{ }^{42}$ Meza and co-workers ${ }^{37,42,44,45}$ and Bauer et al. ${ }^{40}$ successfully used atomic layer deposition to coat -5-60-nm-thick $\mathrm{Al}_{2} \mathrm{O}_{3}$ and $\mathrm{TiN}$ coatings onto polymer scaffolds, as shown in Figure $4 \mathrm{c}-\mathrm{d}$. After deposition, the initial polymer scaffold was exposed by slicing off two of the six faces of the sample using a focused ion beam and then removed by oxygen plasma etching. ${ }^{37,38,42,44,45}$ The TPL method allows the angled tubes of a nanolattice to have smooth 


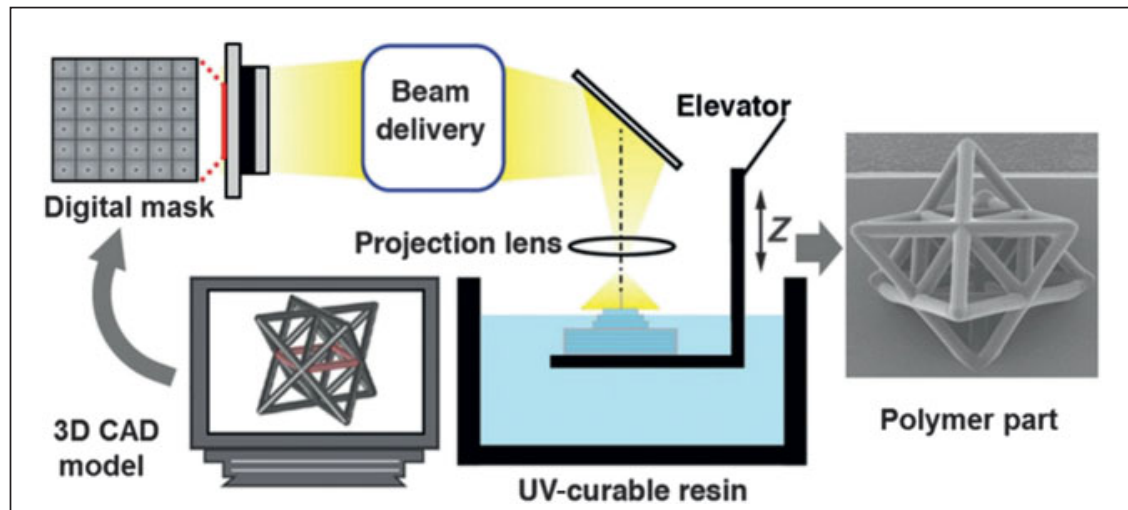

Figure 3. Representative image of a microlattice fabricated by projection microstereolithography, a layer-by-layer technique capable of fabricating arbitrary, microscale, 3D structures with a resolution of $\sim 5 \mathrm{~mm}$. Note: CAD, computer-assisted design. Reproduced with permission from Reference 30. (C) 2014 American Association for the Advancement of Science. failed catastrophically with little to no recovery. ${ }^{38}$ The constituent $\mathrm{Al}_{2} \mathrm{O}_{3}$ is brittle, but as the wall thickness decreases to the nanometer regime, the $\mathrm{Al}_{2} \mathrm{O}_{3}$ contains fewer flaws (e.g., inclusions, voids, and pores) commonly described using Weibull statistics. The material size effect manifests itself here as proximity to ideality, allowing the production of higherquality materials, containing very few flaws, at the nanometer scale. This, in turn, allows the structure to experience shell buckling and to recover at appropriate $t / a$ ratios instead of fracturing at a flaw first, as would be the case for thicker-walled structures. ${ }^{38,45}$

Bauer et al. also studied nonhollow $\mathrm{Al}_{2} \mathrm{O}_{3}$ coated polymer nanolattices fabricated using the DLW TPL method. ${ }^{40}$ They showed that most of the nanolattices failed because of surfaces, in contrast to the rough stair-step edges produced by microstereolithography.

The nanolattice struts produced by TPL have elliptical cross sections, where the ellipticity of the tubes decreases with the unitcell angle, an artifact related to the fabrication process. ${ }^{37,41,43,44}$ Hollow gold nanolattices with an octahedral geometry were fabricated by DLW TPL, and their response to uniaxial compression as a function of unit-cell angle and wall thickness revealed that both the strength and stiffness can be increased by an order of magnitude by tuning the geometry for samples of constant relative density, ${ }^{37}$ in contrast to the behavior predicted by cellular-solids theory, which assumes a constant strength and stiffness for a given relative density. ${ }^{8,43}$ This study demonstrated that both geometrical effects, resulting from changes in unit-cell angle, and material size effects, which emerge from the combination of the atomic-level microstructure of the solid constituent with the particular nano-sized wall thickness, can be exploited to elicit novel mechanical properties that would not exist in a larger version of the structure. ${ }^{37}$

Hollow alumina $\left(\mathrm{Al}_{2} \mathrm{O}_{3}\right)$ nanolattices with the octet-truss geometry with relative densities in the range of $\bar{\rho}=10^{-4}-10^{-1}$ recovered up to $98 \%$ of their initial height after uniaxial compressions in excess of $50 \%{ }^{38}$ Their ductile-like deformation and recoverability has been attributed to the ratio between the wall thickness $(t)$ and the semi-major axis $(a)$ of the hollow elliptical strut cross section. When $t / a$ was less than $\sim 0.03$, the nanolattices deformed by shell buckling and recovered after deformation; at greater $t / a$ values, the nanolattices brittle fracture, and the thinner $\mathrm{Al}_{2} \mathrm{O}_{3}$ coatings in the composite structures carried higher tensile stresses than their thicker
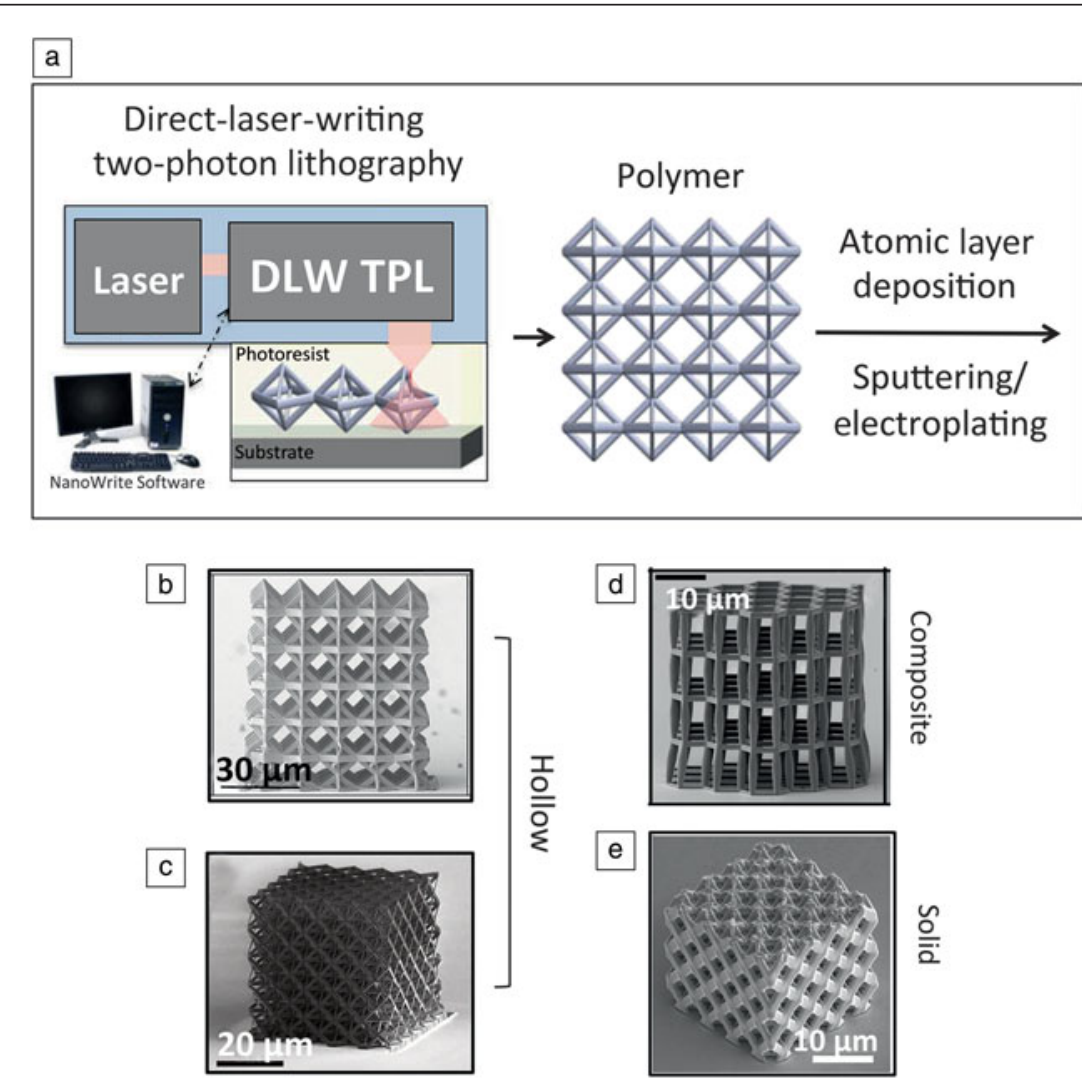

Figure 4. Schematic of (a) direct-laser-writing two-photon lithography (DLW TPL) and (b-e) representative images of nanolattices fabricated by this method: (b) hollow gold, (c) hollow alumina, (d) composite polymer/alumina, and (e) solid copper nanolattices. (a) Reproduced with permission from Reference 42. () 2014 Wiley-Blackwell. (b) Reproduced with permission from Reference 37. (C) 2015 The American Society of Mechanical Engineers. (c) Reproduced with permission from Reference 44. () 2014 American Association for the Advancement of Science. (d) Reproduced with permission from Reference 40. (c) 2014 National Academy of Sciences. (e) Reproduced with permission from Reference 39. (c) 2014 Elsevier. 
counterparts prior to failure. For the thinnest $\mathrm{Al}_{2} \mathrm{O}_{3}$ coatings of $10 \mathrm{~nm}$, microcracks and localized fracture were observed along the strut lengths, and the samples did not fail by fracture of the struts or nodes. ${ }^{40}$ Optimized honeycomb lattice composites, with rigid walls, outperformed existing engineering foams with a comparable density of $1 \mathrm{mg} / \mathrm{cm}^{3}$ by almost an order of magnitude, at compressive strengths of $280 \mathrm{MPa}$ for the $50-\mathrm{nm} \mathrm{Al}_{2} \mathrm{O}_{3}$ case. ${ }^{40}$ Both the hollow and composite $\mathrm{Al}_{2} \mathrm{O}_{3}$ nanolattices achieve new strengths and stiffnesses for their given relative densities while eliciting many novel properties for brittle architected materials, such as recoverability, that result from both material and structural effects. ${ }^{38,40}$

The interplay of structural and material size effects has also been observed in lattices with solid metal struts as the constituent building blocks, created using extensions of the method employed for hollow nanolattices. In contrast to the nanolattices, the smallest length scale of the mesolattice is on the order of microns. ${ }^{39}$ Monolithic (i.e., nonhollow) copper mesolattices with micron-sized struts and unit-cell sizes of 6 and $8 \mu \mathrm{m}$, shown in Figure 4e, were made by first using an inverse DLW TPL method to create a negative of the geometry by patterning in a positive-tone resist and then electroplating the metal into the exposed pores. ${ }^{39}$ The polymer mold was subsequently removed to reveal a mesolattice with solid nodes and struts having diameters on the order of $2 \mu \mathrm{m} .{ }^{39}$ The average grain size for electrochemically deposited copper was $2 \mu \mathrm{m}$, which enabled the grains to span the entire strut diameter.

These mesolattices had relative densities in the range of $\bar{\rho}=0.4-0.8$ and compressive yield strengths of $330 \mathrm{MPa}$, which is up to $80 \%$ higher than that of bulk copper $(133 \mathrm{MPa}){ }^{39}$ For relative densities of 0.8 , strengths of $\sim 330 \mathrm{MPa}$ were observed as a result of the emergence of smaller-is-stronger material size effects in the micron-sized struts. ${ }^{39}$ The mesolattice with architected small-scale copper struts outperformed bulk copper by a factor of up to 2.5 for relative densities of $\bar{\rho}>0.6$, when up to $40 \%$ of the material had been removed. This means that introducing architecture can significantly reduce the weight of the material while simultaneously strengthening it, a finding diametrically opposite to the predictions of classical theories. ${ }^{39}$

In addition to the geometries of structural metamaterials reviewed in this article, many researchers have begun developing and fabricating new geometries with unique properties. Polymer auxetic 3D materials (i.e., materials with a negative Poisson's ratio) have been fabricated using the DLW TPL method. ${ }^{46}$ Upon compression, typical materials expand in directions orthogonal to the loading axis; auxetic materials contract in these directions. ${ }^{46}$ Pentamode materials - $3 \mathrm{D}$ solid materials that, in principle, behave as fluids - have also been designed and fabricated with the TPL approach and provide novel properties in their unique ability to decouple the bulk and shear moduli. ${ }^{47}$ This removes the coupling of shear and compression waves that can travel through a medium. ${ }^{47}$ Both the auxetic and pentamode architectures have unique material properties that support their use in a variety of applications ranging from optical to structural systems. ${ }^{46,47}$

\section{Nonstructural applications of architected materials \\ Photonic crystals: Optical properties of nano- and microarchitected materials}

Three-dimensional photonic crystals ( $\mathrm{PhCs}$ ) are structures whose constituent materials exhibit a periodic variation in the refractive index in all three spatial dimensions. Because of this periodicity, certain $3 \mathrm{D}$ PhCs exhibit a full photonic bandgap (PBG), a range of frequencies for which incident light cannot propagate in any direction. ${ }^{48,49}$ Other $3 \mathrm{D} \mathrm{PhCs}$ have pseudo-stopbands that forbid light propagation in some, not all, crystallographic directions. ${ }^{50}$ The functionalities of 3D PhCs can be divided into two categories: (1) static PhCs, which can guide, ${ }^{51,52}$ diffract, ${ }^{53}$ trap, ${ }^{54}$ and amplify ${ }^{55}$ light through structural design and the introduction of defects, and (2) dynamic PhCs, which can change the frequency of light they reflect by manipulation of their refractive index or periodic dimensions through chemical ${ }^{56}$ or physical $^{57}$ stimuli.

Designed defects, such as lines of modified elements that form waveguides, are necessary to support optical modes and allow for the propagation of frequencies that cannot otherwise exist in the surrounding PBG material, a crucial step toward the realization of PhC-based optical circuitry. ${ }^{51}$ Wegener and co-workers pioneered the use of DLW TPL and double inversion to generate "woodpile" $\mathrm{PhC}$ architectures that contain defect cavities and act as resonators. ${ }^{58}$ Direct laser writing also enables the fabrication of arbitrarily complex micro- and nanostructures and has been exploited to generate chiral $\mathrm{PhCs}$ with polarization stopbands ${ }^{59}$ and $3 \mathrm{D}$ photonic quasicrystals ${ }^{60}$ that exhibit novel photonic properties not available in strictly periodic structures.

Fabrication and characterization of tunable $3 \mathrm{D} \mathrm{PhCs}$ is an area of increasing research interest. Most such structures are based on colloidal crystal arrays, although block copolymers have also been used because self-assembly can yield doublegyroid microdomain architectures under specific chemical conditions. ${ }^{61}$ Inverse-opal structures represent the most common type of 3D tunable PhCs. Arsenault et al. demonstrated an elastomeric inverse-opal structure capable of being reversibly tuned in the optical range when mechanically stretched..$^{50}$ Tunable inverse-opal PhCs have also been generated using various thermosensitive and chemosensitive materials. For example, Aizenberg and co-workers exploited the structural color inherent in silica inverse opals to develop sensors whose optical properties change in different solvents. ${ }^{62}$

\section{Phononic crystals: Acoustic properties of nano- and microarchitected materials}

Similarly to light in photonic crystals, mechanical waves, or phonons, can be modified in materials with periodically varying mechanical properties. ${ }^{63,64}$ This spatial periodicity causes interference between transmitted and reflected waves having 

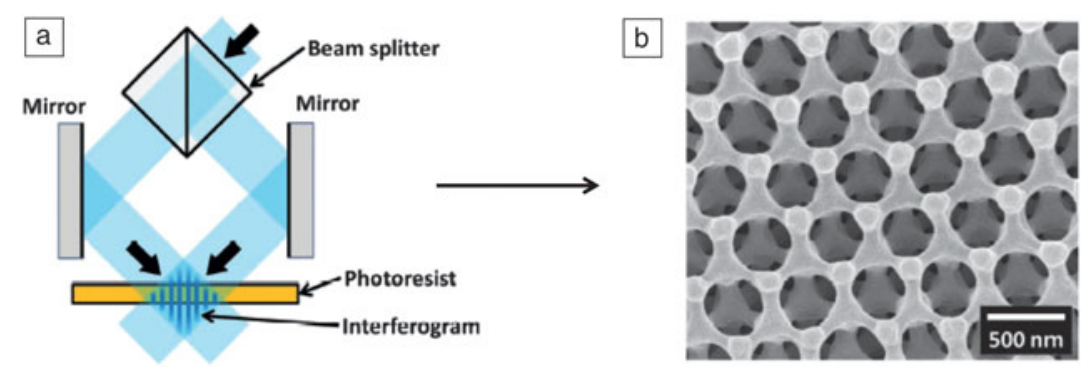

Figure 5. (a) Schematic of multibeam interference lithography and (b) representative scanning electron microscope image of a 3D polydimethylsiloxane/air phononic crystal structure fabricated via interference lithography template. (a) Reproduced with permission from Reference 63. (c) 2012 Wiley-VCH. (b) Reproduced with permission from Reference 74. (C) 2006 American Chemical Society.

wavelengths comparable to the periodicity of the structure and, consequently, alters the propagation of these phonons. ${ }^{62,64}$ Structures that can manipulate phonons in this way are PnCs, and micro- and nanocomposites have been shown to create artificial acoustic dispersion and PnBGs similar to the PBGs in PhCs. ${ }^{65}$

A number of unconventional phenomena can arise from phononic metamaterials, such as acoustic cloaking, ${ }^{66}$ wave filtering, ${ }^{67,68}$ and acoustic damping. ${ }^{69}$ Two-dimensional PnCs have been fabricated with PnBGs in the sonic ${ }^{70}$ (midgap frequency of $\left.10^{2}-10^{3} \mathrm{~Hz}\right)$, ultrasonic $\mathrm{c}^{71}\left(10^{4}-10^{6} \mathrm{~Hz}\right)$, and even hypersonic ${ }^{72}$ $\left(10^{9}-10^{12} \mathrm{~Hz}\right)$ ranges. Fabricating $3 \mathrm{D}$ PnCs with submicron periodicities to control high-frequency phonons with frequencies above $10^{12} \mathrm{~Hz}$ remains a challenge.

Recently, 3D hypersonic PnCs were realized with polymer microspheres self-assembled into opal structures, ${ }^{73}$ and 3D mechanically tunable elastomeric PnCs with 980-nm lattice spacing were fabricated using interference lithography templating $^{74}$ (Figure 5). The submicron periodicity of these PnCs is comparable to the wavelengths of visible and nearinfrared light, implying that these crystals can exhibit bandgaps for both hypersound and light. Akimov et al. used hypersound to modulate the period of a synthetic opal structure, consequently changing the spectral position and width of the structure's photonic stopband - a demonstration of coupled photonic and phononic interactions. ${ }^{75}$ Continued reduction of $\mathrm{PnC}$ periodicity to the nanometer scale promises to extend these structures to the terahertz regime, so that heat transport can be managed in a manner similar to photons and phonons. ${ }^{76}$

\section{Electrochemical devices based on 3D architectures}

Three-dimensional periodically architected materials have been used to increase the efficiency of dye-sensitized solar cells (DSSCs). ${ }^{1,77-79}$ Such materials can be porous, for sensitization and electrolyte infiltration, and can also improve light trapping. Properties of PhCs, including Bragg diffraction, $\mathrm{PBG}$ reflection, multiple scattering in disordered regions, and resonant modes, can greatly enhance the optical light path within the organic-dye active layer of DSSCs. This gives photons a higher probability of being absorbed in this layer, ${ }^{77-79}$ improving an efficiency that is limited, in part, because the organic dyes used in DSSCs are poor absorbers of red and near-infrared light. ${ }^{80}$ Mihi et al. ${ }^{78}$ increased DSSC power efficiency by using a porous PhC coupled to the working electrode. Guldin et al. ${ }^{79}$ achieved similar results by fabricating a double-layer DSSC using titania inverse opals and mesoscopic block-copolymer-patterned titania. This structure allows for effective dye sensitization, electrolyte infiltration, and charge collection from both the mesoporous and $\mathrm{PhC}$ layers.

The nano- and microscale constructs of architected materials have benefits beyond photonic properties for proposed silicon electrodes in lithium-ion batteries. ${ }^{81,82}$ (For more information on lithium-ion batteries, see the article in this issue by Crabtree et al.) Esmanski and Ozin ${ }^{81}$ and Pikul et al ${ }^{83}$ extensively explored silicon inverse opals (Figure 6), and Xia and co-workers ${ }^{82}$ fabricated silicon nanolattices to study how macroporosity and architecture aid in accommodating the dramatic volume swings experienced by the silicon electrode as a result of lithium intercalation during cycling. ${ }^{81}$ The introduction of $3 \mathrm{D}$ architecture into silicon electrodes promises

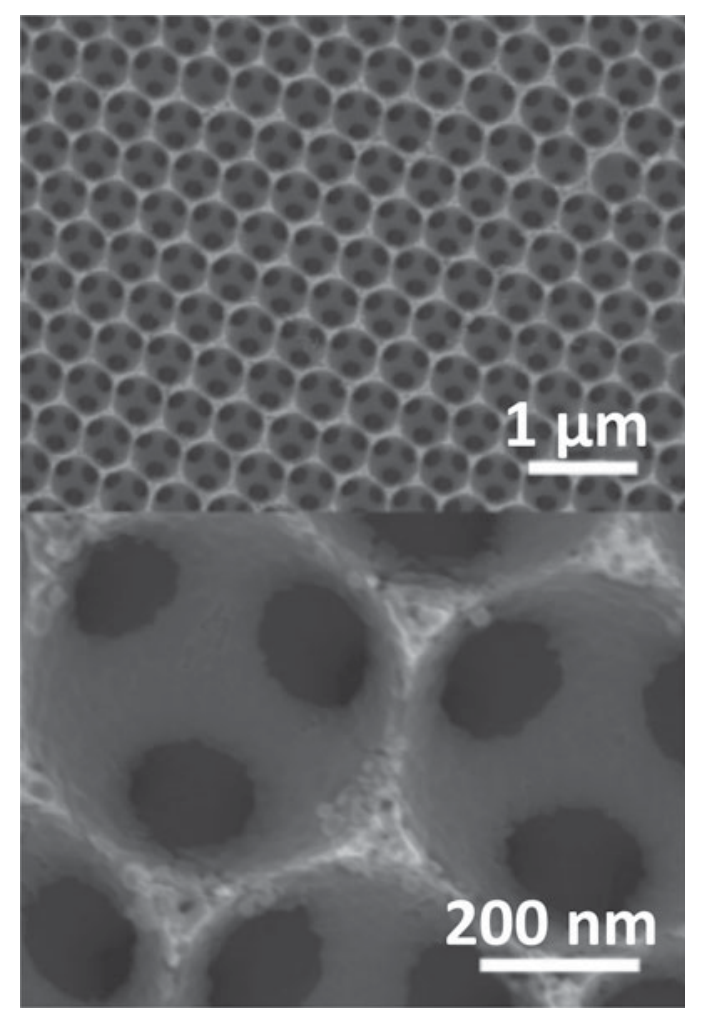

Figure 6. Representative scanning electron microscope images at different magnifications of nanocrystalline silicon inverse opals templated from 480-nm silica spheres for use as negative electrodes in lithium-ion batteries. Reproduced with permission from Reference 81. (C) 2009 Wiley-VCH. 


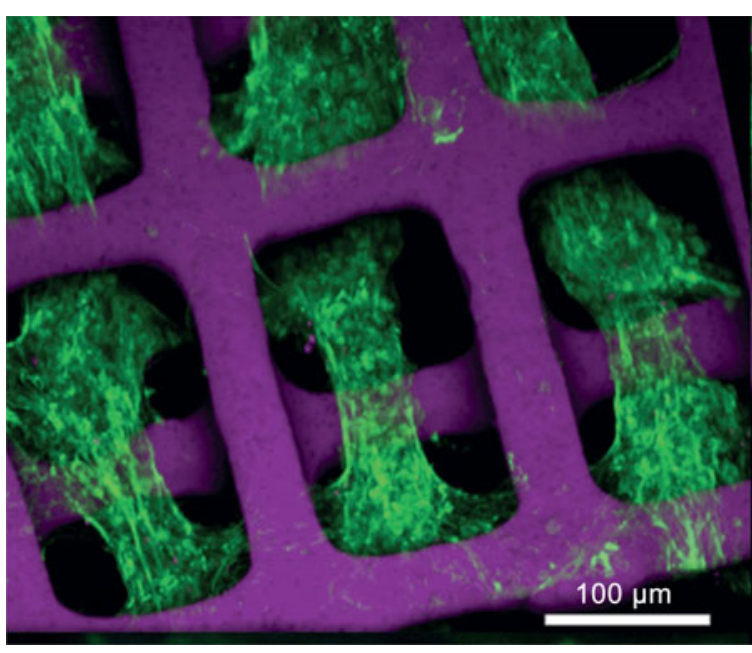

Figure 7. Representative confocal micrograph of neonatal rat heart cells cultured on multilayer poly(glycerol sebacate) scaffolds. Reproduced with permission from Reference 88. (c) 2013 Wiley-VCH.

many advantages, including enhanced mechanical stability, minimal lithium diffusion distances and large surface areas for improved charge and discharge rate, control over porosity for superior electrolyte infiltration and increased cell capacity, and better electrical conductivity than can be obtained with electrodes based on particle aggregates requiring binders and additives. $^{81,82}$

\section{D architected scaffolds for tissue growth}

Human tissues are complex 3D structures that consist of cells and an extracellular matrix in which hierarchy can be found at multiple length scales. Tissue engineering is a growing field, and a great deal of research has focused on controlling cell alignment, differentiation, and proliferation and, eventually, tissue growth. ${ }^{84}$ Aside from chemical cues, cell growth is highly dependent on substrate architecture, with physical properties such as geometry, pore size and connectivity, porosity, and stiffness playing significant roles in determining tissue development. ${ }^{85-87}$

Architected materials allow for precise control over the microscale and mesoscale pore interconnectivity patterns of multilayered scaffolds, which can guide the alignment of cells into multicellular bundles - and eventually into tissues - and can provide mechanical cues for cell differentiation. ${ }^{88}$ In an effort to identify optimal conditions for bone-tissue growth, Sun et al. fabricated rigid silk/hydroxyapatite scaffolds with pore sizes in the range of $200-750 \mu \mathrm{m}$ and assessed the differentiation and growth of human-bone-marrow-derived mesenchymal stem cells (hMSCs) on these substrates. ${ }^{84}$ They found that hMSCs successfully grew onto and into the printed surfaces and organized into swirl-like structures resembling the cell orientation found in canals of human long bone. ${ }^{84}$ Analogously, Kolewe et al. showed how neonatal rat heart cell attachment, elongation, and orientation can be dictated by altering the alignment of scaffold layers, the width of scaffold struts, and the gaps between offset struts (Figure 7). ${ }^{88}$ These examples show that architected scaffold materials are useful not only for understanding cellular interactions with complex 3D environments, but potentially for generating functional tissues.

\section{Outlook and scalable fabrication techniques}

This selective overview highlights some recent structural metamaterials and other architected materials with dimensions that span microns to nanometers. Small-scale experiments have demonstrated that unique material size effects can effectively be propagated onto macroscopic dimensions and, as a result, have the potential to create new classes of engineering materials. ${ }^{37-39}$ Use of architectural features as key elements in defining multidimensional material design spaces promises to enable independent manipulation of historically coupled physical attributes to develop materials with unprecedented capabilities. Some realistic technological advances that would be enabled by incorporating architected metamaterials include materials with tunable mechanical properties, tunable filters and laser sources, tissue implants generated on biodegradable scaffolds that can be directly inserted into the human body, battery-powered implantable chemical sensors, and extremely insulating and superthin thermal linings for jackets and sleeping bags.

The most significant obstacle today to incorporating such metamaterials in useful technological applications is scalability (i.e., manufacturing either a large number of small-scale components or materials with large dimensions in a reasonable amount of time). Some of the possible technologies that could lead to a scalable route to producing small-featured metamaterials include roll-to-roll fabrication with nano-imprintable patterns, holographic lithography, and phase-shifting masks.

\section{References}

1. G. von Freymann, V. Kitaev, B.V. Lotsch, G.A. Ozin, Chem. Soc. Rev. 42, 2528 (2013).

2. J. Sun, B. Bhushan, J. Tong, RSC Adv. 3, 14862 (2013).

3. J. Wang, A.G. Evans, K. Dharmasena, H.N.G. Wadley, Int. J. Solids Struct. 40, 6981 (2003).

4. H. Wadley, Compos. Sci. Technol. 63, 2331 (2003).

5. S. Chiras, D.R. Mumm, A.G. Evans, N. Wicks, J.W. Hutchinson, K. Dharmasena, H.G.N. Wadley, S. Fichter, Int. J. Solids Struct. 39, 4093 (2002).

6. V. Deshpande, N. Fleck, Int. J. Solids Struct. 38, 6275 (2001).

7. V.S. Deshpande, M.F. Ashby, N.A. Fleck, Acta Mater. 49, 1035 (2001)

8. N.A. Fleck, V.S. Deshpande, M.F. Ashby, Proc. R. Soc. Lond. A 466, 2495 (2010).

9. V.S. Deshpande, N.A. Fleck, M.F. Ashby, J. Mech. Phys. Solids 49, 1747 (2001).

10. R.G. Hutchinson, N.A. Fleck, J. Mech. Phys. Solids 54, 756 (2006).

11. D.D. Symons, N.A. Fleck, J. Appl. Mech. 75, 051011 (2008).

12. N.A. Fleck, X. Qiu, J. Mech. Phys. Solids 55, 562 (2007).

13. A.T. Jennings, M.J. Burek, J.R. Greer, Phys. Rev. Lett. 104, 135503 (2010).

14. J.R. Greer, J.T.M. De Hosson, Prog. Mater. Sci. 56, 654 (2011).

15. J.R. Greer, W.D. Nix, Appl. Phys. A 80, 1625 (2005).

16. J.R. Greer, W.C. Oliver, W.D. Nix, Acta Mater. 53, 1821 (2005).

17. R. Dou, B. Derby, Scr. Mater. 61, 524 (2009).

18. M. Dietiker, S. Buzzi, G. Pigozzi, J.F. Löffler, R. Spolenak, Acta Mater. 59, 2180 (2011).

19. C.A. Volkert, E.T. Lilleodden, Philos. Mag. 86, 5567 (2006). 
20. S.H. Oh, M. Legros, D. Kiener, G. Dehm, Nat. Mater. 8, 95 (2009).

21. J.R. Greer, D. Jang, X.W. Gu, J. Mater. 64, 1241 (2012).

22. X.W. Gu, C.N. Loynachan, Z. Wu, Y.-W. Zhang, D.J. Srolovitz, J.R. Greer, Nano Lett. 12, 6385 (2012).

23. B. Yang, C. Motz, M. Rester, G. Dehm, Philos. Mag. 92, 3243 (2012).

24. J. Rys, L. Valdevit, T.A. Schaedler, A.J. Jacobsen, W.B. Carter, J.R. Greer, Adv. Eng. Mater. 16, 889 (2014).

25. D.Z. Chen, D. Jang, K.M. Guan, Q. An, W.A. Goddard, J.R. Greer, Nano Lett. 13, $4462(2013)$

26. C.Q. Chen, Y.T. Pei, J.T.M. De Hosson, Acta Mater. 58, 189 (2010).

27. C.A. Volkert, A. Donohue, F. Spaepen, J. Appl. Phys. 103, 083539 (2008)

28. A.M. Hodge, J. Biener, L.L. Hsiung, Y.M. Wang, A.V. Hamza, J.H. Satcher, J. Mater. Res. 20, 554 (2005).

29. A.J. Jacobsen, W. Barvosa-Carter, S. Nutt, Adv. Mater. 19, 3892 (2007).

30. X. Zheng, H. Lee, T.H. Weisgraber, M. Shusteff, J. DeOtte, E.B. Duoss,

J.D. Kuntz, M.M. Biener, Q. Ge, J.A. Jackson, S.O. Kucheyev, N.X. Fang,

C.M. Spadaccini, Science 344, 1373 (2014)

31. J. Fischer, M. Wegener, Laser Photon. Rev. 7, 22 (2013).

32. H.-B. Sun, S. Kawata, Adv. Polym. Sci. 170, 169 (2004)

33. W. Xiong, Y.S. Zhou, X.N. He, Y. Gao, M. Mahjouri-Samani, L. Jiang, T. Baldacchini, Y.F. Lu, Light Sci. Appl. 1, e6 (2012).

34. A.J. Jacobsen, W. Barvosa-Carter, S. Nutt, Acta Mater. 56, 2540 (2008).

35. T.A. Schaedler, A.J. Jacobsen, A. Torrents, A.E. Sorensen, J. Lian, J.R. Greer, L. Valdevit, W.B. Carter, Science 334, 962 (2011).

36. A. Torrents, T.A. Schaedler, A.J. Jacobsen, W.B. Carter, L. Valdevit, Acta Mater. 60, 3511 (2012).

37. L.C. Montemayor, J.R. Greer, J. Appl. Mech. 82, 071012 (2015).

38. L.R. Meza, S. Das, J.R. Greer, Science 345, 1322 (2014).

39. X.W. Gu, J.R. Greer, Extreme Mech. Lett. 2, 7 (2015).

40. J. Bauer, S. Hengsbach, I. Tesari, R. Schwaiger, O. Kraft, Proc. Natl. Acad. Sci. U.S.A. 111, $2453(2014)$

41. L. Valdevit, S.W. Godfrey, T.A. Schaedler, A.J. Jacobsen, W.B. Carter, J. Mater. Res. 28, 2461 (2013).

42. L.C. Montemayor, L.R. Meza, J.R. Greer, Adv. Eng. Mater. 16, 184 (2014).

43. L.J. Gibson, M.F. Ashby, Cellular Solids: Structure and Properties (Cambridge University Press, Cambridge, UK, 1999).

44. L.R. Meza, J.R. Greer, J. Mater. Sci. 49, 2496 (2014).

45. D. Jang, L. Meza, F. Greer, J. Greer, Nat. Mater. 12, 893 (2013).

46. T. Bückmann, N. Stenger, M. Kadic, J. Kaschke, A. Frölich, T. Kennerknecht, C. Eberl, M. Thiel, M. Wegener, Adv. Mater. 24, 2710 (2012).

47. M. Kadic, T. Bückmann, N. Stenger, M. Thiel, M. Wegener, Appl. Phys. Lett. 100, 191901 (2012)

48. A. Blanco, E. Chomski, S. Grabtchak, M. Ibisate, S. John, S. Leonard, C. Lopez, F. Meseguer, H. Miguez, J. Mondia, G. Ozin, O. Toader, H.M. van Driel, Nature 405, $437(2000)$.

49. S. Noda, K. Tomoda, N. Yamamoto, A. Chutinan, Science 289, 604 (2000).

50. A.C. Arsenault, T.J. Clark, G. von Freymann, L. Cademartiri, R. Sapienza,

J. Bertolotti, E. Vekris, S. Wong, V. Kitaev, I. Manners, R.Z. Wang, S. John,

D. Wiersma, G.A. Ozin, Nat. Mater. 5, 179 (2006)

51. S.A. Rinne, F. García-Santamaría, P.V. Braun, Nat. Photonics 2, 52 (2007)

52. M. Deubel, M. Wegener, S. Linden, G. von Freymann, S. John, Opt. Lett. 31, 805 (2006).

53. T. Maka, D.N. Chigrin, S.G. Romanov, C.M. Sotomayor Torres, Prog. Electromagn. Res. 41, 307 (2003).

54. J-Y. Chen, Physica E 44, 43 (2011)

55. Y.A. Vlasov, K. Luterova, I. Pelant, B. Hönerlage, V.N. Astratov, Appl. Phys. Lett. 71, 1616 (1997)

56. A.C. Arsenault, D.P. Puzzo, I. Manners, G.A. Ozin, Nat. Photonics 1, 468 (2007).

57. H. Fudouzi, T. Sawada, Langmuir 22, 1365 (2006).

58. G. von Freymann, A. Ledermann, M. Thiel, I. Staude, S. Essig, K. Busch, M. Wegener, Adv. Funct. Mater. 20, 1038 (2010).

59. M. Thiel, M. Decker, M. Deubel, M. Wegener, S. Linden, G. von Freymann, Adv. Mater. 19, 207 (2007).

60. A. Ledermann, L. Cademartiri, M. Hermatschweiler, C. Toninelli, G.A. Ozin, D.S. Wiersma, M. Wegener, G. von Freymann, Nat. Mater. 5, 942 (2006).

61. A.M. Urbas, M. Maldovan, P. DeRege, E.L. Thomas, Adv. Mater. 14, 1850 (2002).

62. I.B. Burgess, L. Mishchenko, B.D. Hatton, M. Kolle, M. Lončar, J. Aizenberg, J. Am. Chem. Soc. 133, 12430 (2011).

63. J-H. Lee, J.P. Singer, E.L. Thomas, Adv. Mater. 24, 4782 (2012).

64. M. Maldovan, Nature 503, 209 (2013).

65. M. Kushwaha, P. Halevi, L. Dobrzynski, B. Djafari-Rouhani, Phys. Rev. Lett. 71, 2022 (1993)

66. S. Zhang, C. Xia, N. Fang, Phys. Rev. Lett. 106, 024301 (2011).

67. Z. Liu, X. Zhang, Y. Mao, Y.Y. Zhu, Z. Yang, C.T. Chan, P. Sheng, Science 289, 1734 (2000).
68. K.M. Ho, C.K. Cheng, Z. Yang, X.X. Zhang, P. Sheng, Appl. Phys. Lett. 83, 5566 (2003).

69. S. Babaee, J. Shim, J.C. Weaver, E.R. Chen, N. Patel, K. Bertoldi, Adv. Mater. 25, 5044 (2013).

70. J. Vasseur, P. Deymier, B. Chenni, B. Djafari-Rouhani, L. Dobrzynski, D. Prevost, Phys. Rev. Lett. 86, 3012 (2001)

71. F. Montero de Espinosa, E. Jiménez, M. Torres, Phys. Rev. Lett. 80, 1208 (1998).

72. T. Gorishnyy, C. Ullal, M. Maldovan, G. Fytas, E. Thomas, Phys. Rev. Lett. 94, 115501 (2005).

73. W. Cheng, J. Wang, U. Jonas, G. Fytas, N. Stefanou, Nat. Mater. 5, 830 (2006)

74. J.-H. Jang, C.K. Ullal, T. Gorishnyy, V.V. Tsukruk, E.L. Thomas, Nano Lett. 6. $740(2006)$

75. A. Akimov, Y. Tanaka, A. Pevtsov, S. Kaplan, V. Golubev, S. Tamura, D. Yakovlev, M. Bayer, Phys. Rev. Lett. 101, 033902 (2008).

76. M. Maldovan, Phys. Rev. Lett. 110, 025902 (2013)

77. S. Nishimura, N. Abrams, B.A. Lewis, L.I. Halaoui, T.E. Mallouk, K.D. Benkstein, J. van de Lagemaat, A.J. Frank, J. Am. Chem. Soc. 125, 6306 (2003).

78. A. Mihi, C. Zhang, P.V. Braun, Angew. Chem. Int. Ed. 50, 5712 (2011).

79. S. Guldin, S. Hüttner, M. Kolle, M.E. Welland, P. Müller-Buschbaum, R.H. Friend, U. Steiner, N. Tétreault, Nano Lett. 10, 2303 (2010).

80. B. O'Regan, M. Grätzel, Nature 353, 737 (1991)

81. A. Esmanski, G.A. Ozin, Adv. Funct. Mater. 19, 1999 (2009).

82. X. Xia, W. Gu, J. Greer, "Fabrication and In Situ SEM of Mechanically Robust Si Nano-Trusses as Li Battery Electrodes," presented at the 2014 MRS Fall Meeting, Boston, December 1-4, 2014.

83. J.H. Pikul, H. Gang Zhang, J. Cho, P.V. Braun, W.P. King, Nat. Commun. 4, 1732 (2013).

84. L. Sun, S.T. Parker, D. Syoji, X. Wang, J.A. Lewis, D.L. Kaplan, Adv. Healthcare Mater. 1, 729 (2012).

85. M. Rumpler, A. Woesz, J.W.C. Dunlop, J.T. van Dongen, P. Fratzl, J. R. Soc. Interface 5, 1173 (2008).

86. M. Fröhlich, W.L. Grayson, L.Q. Wan, D. Marolt, M. Drobnic, G. VunjakNovakovic, Curr. Stem Cell Res. Ther. 3, 254 (2008).

87. V. Karageorgiou, D. Kaplan, Biomaterials 26, 5474 (2005)

88. M.E. Kolewe, H. Park, C. Gray, X. Ye, R. Langer, L.E. Freed, Adv. Mater. 25, 4459 (2013).

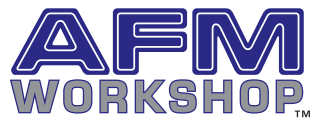

\section{American Ingenuity}
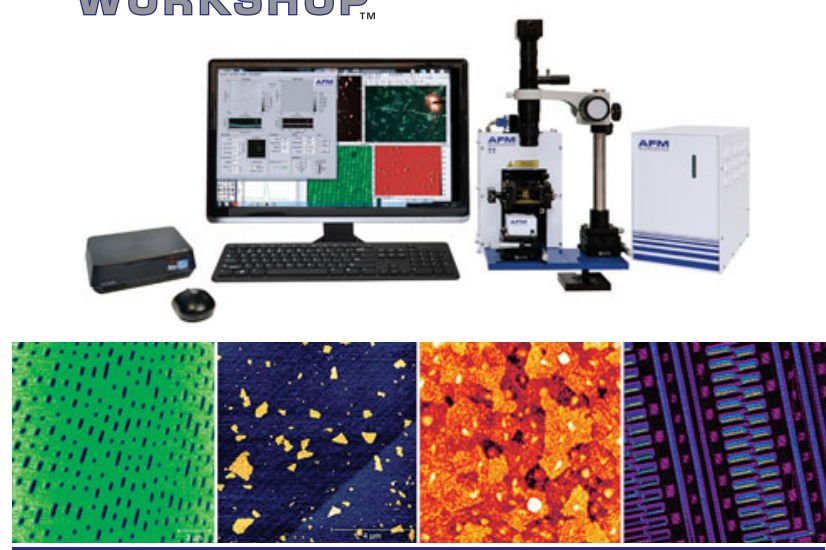

ATOMIC FORCE MICROSCOPES from AFMWorkshop

Highest Performing AFMs for the Lowest Cost

Full Systems from $\$ 26,450$

Noise Floor $<.08 \mathrm{~nm}$

1434 E $33^{\text {rd }}$ St., Signal Hill, CA 90755 www.afmworkshop.com | 888-671-5539 